\title{
SOFTWARE TOOLS FOR ENGINEERING DARK SKIES
}

\author{
A. M. SMITH, and \\ D. L. DILAURA, FIES \\ Lighting Technologies, Inc., 2540 Frontier, Suite 107 \\ Boulder, Colorado 80303 U.S.A.
}

\begin{abstract}
Atmospheric scattering of outdoor nighttime electric illumination produces the principal component of background sky luminance that seriously affects ground-based optical astronomy. The sources for this scattering are light emitted skyward directly from luminaires, and light reflected off the ground and other illuminated objects. Careful illumination engineering can thus significantly reduce background sky luminance in two ways: 1 ) by providing outdoor electric lighting equipment that controls the directions in which light is emitted, and; 2) by proper design of outdoor lighting systems which make efficient use of the least amount of light. Recent developments in applied mathematics and computer software have produced computational tools that are being used to design lighting equipment and lighting systems. The software system for luminaire design significantly reduces the cost of this process by eliminating the need for extensive prototyping and provides for inexpensive experimentation with new designs. The system for outdoor lighting calculations permits the design of highly controlled lighting systems that eliminate glare and upward directed light while providing light appropriate for the visual task. These two software systems are described, along with examples of their use in areas that directly affect astronomical observations.
\end{abstract}

\section{INTRODUCTION: ENGINEERING DARK SKIES}

Outdoor lighting is generally considered beneficial in increasing the safety of roadways and the security of homes and public places. An unavoidable result of outdoor lighting is light directed into the atmosphere which scatters off particles; thereby raising the background luminance of the sky. High background sky luminance is detrimental to optical astronomy, because it masks objects fainter than the luminance of the sky itself. Ironically, as sky luminance has grown in recent years, so has the need to study ever fainter objects.

Light directed into the atmosphere comes from reflection off illuminated surfaces and directly from the luminaire. It is possible to satisfy nighttime lighting needs while minimizing the amount of light into the atmosphere. This is done by providing only as much light on illuminated surfaces as is actually needed for the visual task, and by preventing light from leaving the luminaire in any direction other than toward the visual task. 
There is no doubt that, except at a few sites, background nighttime sky luminance has increased greatly. These exceptions are at locations where astronomers, users, and municipal governments understand and control the practical forces responsible for the type and amount of outdoor lighting installed.

The four forces which influence the ultimate form of outdoor lighting are political forces, market forces, cost, and technology. Political forces are manifest in the form of lighting ordinances, designed to enforce minimal standards of lighting system performance. Ordinances that are effective must be practical and enforceable. Practicality is determined in part by the ability of a lighting system designer to predict compliance; enforceability is constrained by the types of equipment available. Market forces are reflected in the types of equipment that lighting manufacturers are able to profitably design, fabricate, and sell. Market forces are defined by the equilibrium established between consumer desires and technical feasibility. Cost is borne by the owner of the lighting system, who must not only pay for the initial and life cycle cost of the system, but is also liable for its performance. Technology has a strong influence on the other three forces. The technology of designing lighting equipment and lighting systems has enjoyed tremendous growth in the past five years.

The key to conserving the night sky is to realign these forces to support the design of lighting systems that treat the control of light as an important criteria. The software tools described here will make this possible, because they can be used to demonstrate that careful control of light is enforceable, marketable, and cost-effective.

\section{DEFINING NIGHTTIME LIGHTING NEEDS}

Applications for nighttime illumination include: safe driving conditions, safe public spaces, security of private facilities, and merchandising. All of these require relatively small amounts of carefully placed light. Much of the light which causes high background sky luminance is unused for the intended visual function, and can be eliminated without compromise. In almost all cases, this is light that is not directed to the surfaces intended to be illuminated. Lighting is often not needed through the entire night, and in many cases the amount of light required in the second half of the night is significantly less than that needed in the first half.

\section{FINDING SOLUTIONS THAT SATISFY LIGHTING AND ASTRONOMY REQUIREMENTS}

The process of finding solutions to nighttime lighting needs involves the manufacturers of equipment, the engineer/designer that specifies it, and the owner who operates it. The design process usually follows five steps:

1. information is gathered about the visual task,

2. this information is used to establish design criteria,

3. the design criteria are translated into the desired lighting effect,

4. available equipment is chosen to achieve desired effect,

5. a lighting system design is formulated. 
Lighting professionals are already aware of lighting needs for the performance of nighttime visual tasks. Education is making them aware of the need for dark skies. The technology is now available to make elimination of stray light a design criterion; lighting ordinances can set strict requirements for stray light control, because new analytic tools make such control feasible.

An important component of this process is the availability of good lighting equipment. Commercially available equipment is the result of a design process that has hitherto been so difficult and expensive that only a few criteria could be considered. Most manufacturers have not had the resources to redesign their product line and improve their luminaires or explore new ideas and new light sources. The need for a particular quantity of light at the site of the visual task has always ranked much higher in importance than the need for control. New computational tools now make it possible to reduce the difficulties and diminish design costs, permitting a consideration of a broader set of design criteria.

An equally important component of this process is the integration of luminaires into a system design. This is a matter of deciding the placement and quantity of light needed. It has been difficult for the illumination engineer to be certain that a proposed solution will fulfill the criteria. The usual response to this uncertainty is to overdesign, abandoning careful and conservative use of light in order to be certain that nighttime visual needs are satisfied.

The availability of fast and accurate computer programs allows the engineer to analyze a proposed design with a high degree of certainty. This provides the confidence to abandon the practice of overdesign, thus using less light. The result is less light used more effectively, producing less scatter, and less energy waste.

\section{SOFTWARE TOOLS FOR ENGINEERING BETTER LIGHTING EQUIPMENT}

Most outdoor luminaires satisfy nighttime vision needs, when used as intended. Many of these luminaires, however, exhibit poor control characteristics. Poor control is manifest as unnecessary uplight, glare, and wasted energy.

In almost all cases, light that is directed upward from outdoor luminaires is of no use. This wasted light is a prime source of background sky luminance. Glare is excessive luminance contrast and is commonly encountered as a bright luminaire against a dark outdoor surround. Glare is indicative of poor control; if an observer sees that much light, it is probably not directed to the surfaces that are intended to be illuminated and is contributing to upward directed scatter. Glare is a hazard and a nuisance as well. Quality lighting equipment is characterized by precise control of the directions into which the luminaire distributes light. This characteristic, called cutoff, prevents the luminaire from being seen when viewed from directions away from the visual task.

Energy used to generate light that is directed anywhere but to the site is wasted energy. It is easy to show that the cost of this wasted energy over the lifetime of the luminaire exceeds the cost of designing and manufacturing equipment that is precisely controlled and not wasteful. 


\section{HOW BETTER LIGHTING EQUIPMENT IS DESIGNED}

The process of designing a luminaire takes place in several stages:

1. performance criteria are established,

2. a performance concept is generated (where light is to be placed),

3. the optical system is designed,

4. the optical design is prototyped,

5. the prototype is tested and evaluated,

6. the optical design is modified (return to step 4).

In establishing performance criteria, the luminaire designer identifies the performance characteristics which are desired by customers. Efficiency and quantity of light rank high; cutoff and glare usually rank low. Drawing on a largely empirical knowledge of lighting optics, the engineer designs an optical system to satisfy the performance concept.

The optical design is then sent to the shop, where skilled labor tediously constructs a working model of the design. This is costly in terms of labor, time, and materials. The prototype is photometrically tested to verify compliance with the design criteria.

The first prototype rarely satisfies the design criteria. Once test results are reviewed, the optical design is altered to correct deficiencies. The prototype is modified (or completely rebuilt) and sent for more testing. This cycle is typically executed 6 times.

There are several reasons why this process is difficult. First, the design of non imaging optics is very complex for anything other than a point source, and most electric lamps are not point sources. Second, the detailed directional reflectance characteristics of common reflector materials have not been well understood. Third, very few luminaire designers have a solid theoretical foundation of the physics behind non-imaging optics and luminous radiative transfer.

The result is that most luminaire design is done very empirically, and the cost of the process is very high. This cost, coupled with weak market pressure for innovation, means that new luminaire design projects are not often undertaken. When they are, the list of criteria is kept brief, because with limited analysis tools, it is difficult to optimize many criteria at once. Issues of cutoff, glare control, and uplight are rarely included in the list of criteria.

Recent developments in applied mathematics, combined with advances in micro-computer technology, have made possible a sophisticated luminaire design tool that is revolutionizing the way in which luminaires are designed. The result is that it will become cost effective to design lighting equipment that satisfies nighttime lighting needs with minimum impact on optical astronomy.

FiELD (Finite Element Luminaire Design) is a PC based computer program that incorporates these mathematical and hardware advances. FiELD uses luminous radiative transfer, rather than ray tracing, to describe the exchange of flux between non-imaging optical surfaces.

Using a CAD program, a geometric model of the optical system is constructed. This model is treated as a system of finite elements, each of which exchanges flux with all of the other elements in the system. A system of simultaneous equations is established, which takes into account the geometric 
attributes, and real directional reflectance and transmittance characteristics of the optical surfaces. This system of equations is solved for the steady state condition. The information that results is the complete spatial distribution of light exitant from each element, reported in the form of luminance (candelas / square meter). Since luminance is the most fundamental quantity used in illumination engineering, all other quantities, such as luminous intensity distributions, illuminance, and even predictions of brightness, can be derived from it. Lamps and optical materials are chosen from a library. The luminaire designer requests a report of the desired information, and FiELD generates that information in a short period of time.

Using FiELD, the iterative cycle of luminaire design proceeds much more quickly; deficiencies of the design are corrected in the geometric CAD model, and the resulting change in performance evaluated in a few hours. Elimination of costly prototyping leads to a much more cost effective design process; the list of design criteria can be expanded to include issues of concern to astronomers.

Uplight, glare, and efficiency can be quickly evaluated with the FiELD program. Because the photometric data generated by FiELD is identical to that which is measured for the prototype in the laboratory, the proposed luminaire can be tested with the lighting system analysis program to evaluate the performance in real applications before it even exists. The analytic power of FiELD permits the evaluation and design of luminaires using large sources such as low pressure sodium.

Because the FiELD program makes the process of designing luminaires much more efficient, it can be undertaken with a much smaller investment of resource. This enables small equipment manufacturers to embark on innovative luminaire design projects which result in well controlled equipment that can compete against the larger equipment manufacturers, who have not traditionally been very responsive to the pressure to design better equipment. Good quality equipment can be competitively priced, and it is hoped that competitive pressure will eliminate poor quality equipment from the marketplace.

\section{SOFTWARE TOOLS FOR DESIGNING BETTER LIGHTING SYSTEMS}

A poor lighting system is one which fails to adequately address nighttime vision needs, and/or produces excessive light which contributes to raising background sky luminance.

Under nighttime lighting conditions, small amounts of light, when properly placed, can satisfy visual needs. Excessive amounts of light are often used when the visual task is poorly understood, or the information to quantify lighting system performance is inadequate.

In an attempt to provide adequate light where needed, a "blanket of light" is laid down, providing excessive light even where not needed. This occurs in two ways:

1. In an effort to save money, poor equipment is chosen. Poor equipment can often be used to crudely satisfy immediate visual needs with complete disregard for the needs of astronomy.

2. Equipment which has been carefully designed is misused in a poor lighting system design. This happens when the wrong fixture is used for the job, or the proper fixture is improperly installed. 


\section{Lighting System Analysis Programs}

There are a number of computer programs available to analyze the performance of lighting systems. When of high quality, these programs help the engineer address a long list of criteria quickly and with confidence. High quality programs are characterized by speed, accuracy, and ease of use. One such program is Lumen-Point.

Lumen-Point is an outdoor lighting analysis program that treats luminaires as point sources and uses the inverse square cosine law to compute illuminance at a point. Reflections off illuminated surfaces are not considered. Lumen Point utilizes bicubic splines for reliable interpolation of luminaire candela distributions.

Lumen Point calculates illuminances at points in an arbitrarily oriented plane. These illuminances can be presented as a scaled grid, isocontour plot, or shaded grayscale plot. Statistical information is calculated to evaluate average light levels and uniformity. Lumen Point is also capable of computing roadway luminance and veiling luminance (a metric that quantifies the effect of glare on visibility).

The engineer can evaluate, with arbitrarily fine resolution, the spatial distribution of light on and around the visual task. If the desired distribution of light has been clearly defined, then the compliance of the proposed solution is easily evaluated. Deficiencies in the design are readily located, and modifications to the design can be made in minutes.

In the past, it was not possible to be certain about the performance of lighting systems that required careful control of light. The solution was to simply evenly distribute a generous a mount of light over the site. The certainty purchased with the use of programs such as Lumen Point makes this design approach obsolete. It is now possible to use light only where needed, resulting in energy savings and dark skies, without compromising night time vision requirements. This design certainty is critical for the development of practical outdoor lighting ordinances, since the prediction of compliance is essential if ordinances are to be effective.

\section{CONCLUSIONS}

Nighttime lighting needs can be satisfied without threatening the preservation of dark skies. This is accomplished by carefully considering how much light is needed for the task, and carefully controlling the light used to fill that need. Traditionally, the lighting design process has been more concerned about quantity of light than control of light. To bring about changes in lighting design practice requires that political forces, market pressure, and cost factors be realigned to support the conservative use of light. The technology, in the form of the software tools described here, provides a foundation for such a realignment. It is now possible to raise our expectations about the performance of lighting systems which provide for our lighting needs without endangering the darkness of the night sky. 